\title{
An overview of immunopathogenesis towards COVID-19 infection
}

\author{
Noha Saber Shafik , * Mona Mohammed Abd El Rhman \\ *Department of Medical Microbiology and Immunology, Faculty of Medicine, Sohag \\ University \\ **Department of Tropical Medicine \& Gastroenterology, Faculty of Medicine, Sohag \\ University
}

\begin{abstract}
:
Patients complaining from cough, fever, dyspnea, and severe pneumonia were recorded in Wuhan, China in late December 2019 due to an unknown microbial agent. Virus genome sequencing of the examined patients during this period confirmed the presence of $\beta$ - $\mathrm{CoV}$ strain. This extracted new $\beta$-CoV exhibits 88 percent similarity to the series of 2 severe bat-derived acute respiratory syndromes (SARS)-like coronaviruses, bat-SLCoVZC45, and bat-SL-CoVZXC21, and about 50 percent identical to the MERS-CoV series. Later, the International Commission for Virus Classification called the novel $\beta$ CoV "SARS-CoV-2." On the eleventh of February 2020, the World Health Organization (WHO) reported another name for the disease caused by the 2019-n CoV: which is Corona Virus Disease (COVID-19). Several complex immune system mechanisms exist against the newly discovered COVID-19. Communication has undergone between different branches of the immune system, but the exact mechanism remained unclear until now. This study helps to clarify COVID-19 molecular immune pathogenesis.
\end{abstract}

Keywords: COVID-19, T helper, cytokines, lymphopenia, MHC molecules.

Abbreviations: ARDS: acute respiratory distress syndrome, COVID -19: Coronavirus disease, 2019, MERS: the Middle East respiratory syndrome, RMSD: root mean square deviation, ERGIC: Endoplasmic reticulum-Golgi intermediate compartment, MIP: macrophage inflammatory protein, MBL: mannose-binding lectin, TGF $\beta$ : Transforming growth factor $\beta, \mathrm{MCP}-1$ : monocyte chemoattractant protein, ADE: Antibody-dependent enhancement.

\section{Introduction:}

In December 2019 a coronavirus outbreak was reported in Wuhan City, China, currently known as Coronavirus Novel 2019 (2019-Nov). As of 30 January 2020, China reported that 7,734 cases were infected with COVID-19. ${ }^{(1)}$ The World Health Organization (WHO) stated on February 11, 2020, another name for the 2019-n CoV-caused disease: which is Corona Virus Disease

(COVID-19). At this time, WHO announced the disease as a pandemic. ${ }^{(2)}$ Most coronaviruses are pathogenic to humans. Most of them suffer from mild clinical symptoms, with two serious clinical presentations: severe acute respiratory syndrome (SARS), coronavirus (SARS-CoV), and Middle East respiratory syndrome (MERS). ${ }^{(1,3)}$ Coronaviruses are enveloped viruses having a positive-sense $(26-32 \mathrm{~kb})$ 
An overview of immunopathogenesis towards COVID-19

Noha Saber Shafik single-stranded RNA genome. To date, four genera of coronaviruses $(\alpha, \beta, \pi, \ddot{y})$ which affect human found in the genera $\alpha$ coronavirus (HCoV-229E and NL63) and $\beta$ coronavirus (MERS-CoV, SARS$\mathrm{CoV}, \mathrm{HCoV}-\mathrm{OC} 43$ and, $\mathrm{HCoV}-\mathrm{HKU} 1)$. (4)

Patients with acute respiratory distress syndrome (ARDS) with coughing, fever, and dyspnea due to an unknown microbial agent were found in Wuhan, China, in late December 2019. Sequencing of the virus genome of all patients established the involvement of $\beta-\mathrm{CoV}$ strain. This extracted new $\beta$ $\mathrm{CoV}$ exhibits 88 percent similarity to the sequence of two severe bat-derived acute respiratory syndromes (SARS) like coronaviruses, bat-SL-CoVZC45, and bat-SL-CoVZXC21, and around 50 percent identical to the MERS-CoV strain. The International Virus Classification Commission then named the novel $\beta-\mathrm{CoV}$ "SARS-CoV-2". (5) This study helps to clarify COVID-19 molecular immune pathogenesis.

Morphology of the virus:

Coronaviruses are large pleomorphic spherical particles with a bulbous base, $120 \mathrm{~nm}$ in diameter and $80 \mathrm{~nm}$ in-depth, and $20 \mathrm{~nm}$ in spikes. These viruses were with positive-stranded RNA (Figure 1). ${ }^{(6)}$ The $2019-\mathrm{nCoV}$ is the 7th member of the RNA enveloped coronavirus. ${ }^{(7)}$ The overall SARS-CoV2 ' $S$ ' protein structure is similar to that of SARS-CoV ' $S$ ' with a root- mean square deviation (RMSD) of $3.8 \AA$ above $959 \mathrm{C} \alpha$ atoms. ${ }^{(8)}$ Spike protein (S) aids the binding and entry of SARSCoV-2 into cells. S1 glycoprotein subunit allows tight binding to the ACE2 receptor, whereas, fusion with the target cell as a subunit of S2 is maintained. ${ }^{(9)}$ Within the host, the respiratory tract and gastrointestinal tract epithelial cells can be affected. Coronaviruses typically infect respiratory epithelial cells (pneumocytes), and enteric cells (enterocytes), and cause cytopathic changes. The main targets of COVID 19 are type two pneumocytes and unciliated bronchial epithelial cells. ${ }^{(10)}$ It has revealed cytopathic effects on respiratory epithelial cells and cessation of cilium beating in the lungs. ${ }^{(11)}$

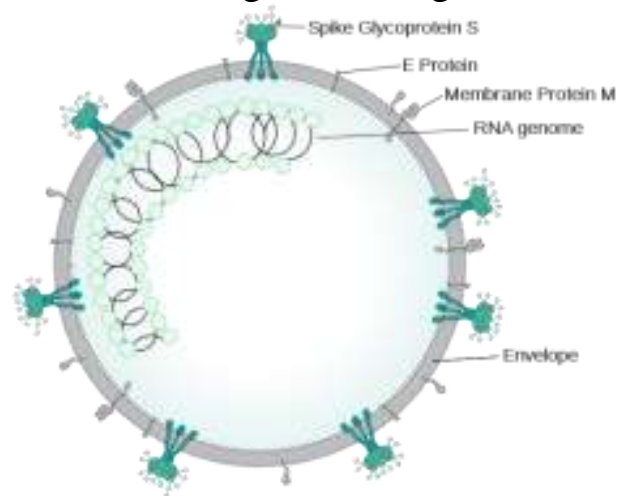

Figure (1): Morphology of coronavirus ${ }^{(6)}$

\section{Pathogenesis of COVID-19:}

COVID-19 infected patients showed radiographic evidence similar to SARS$\mathrm{CoV}$ and MERS-CoV. Also, multiple manifestations such as fever, nonproductive cough, dyspnea, myalgia, fatigue, and pneumonia were reported in all strains. Since the pathogenesis of COVID-19 has not been established, the related mechanisms of SARS-CoV and MERS-CoV can also help us to understand the pathogenesis of SARS$\mathrm{CoV}-2$ infection to promote our knowledge of COVID-19. ${ }^{(12)}$

\section{Coronavirus entry and replication:}

Coronavirus $\mathrm{S}$ protein is a major determinant of the entry of the virus into the target cells. The spike 
An overview of immunopathogenesis towards COVID-19

Noha Saber Shafik glycoprotein envelope attaches to ACE2 for SARS-CoV and SARS-CoV2, CD 209L (a lectin type C, also named L-SIGN) for SARS-CoV, and DPP4 for MERS-CoV. Initially, the passage of SARS-CoV into cells was enhanced through the direct fusion of the membrane between the virus and the plasma membrane. ${ }^{(13)}$

The key proteolytic cleavage event on the SARS-CoV S protein occurred at the location (S2), mediated membrane fusion, and viral infectivity. The MERS-CoV has also produced an irregular two-step activation for membrane fusion. In addition, clathrindependent and independent endocytosis also helps the entry of SARS-CoV. Following the entry of the virus, there is a release of the viral RNA genome is into the cytoplasm and then the translation of this genome into two polyproteins and structural proteins. After, these steps the viral replication starts. ${ }^{(14)}$

The recently developed envelope of glycoproteins attaches to the endoplasmic reticulum or Golgi membrane, and the nucleocapsid is developed by combining genomic RNA and nucleocapsid protein. The viral particles then germinate into the endoplasmic compartment of the intermediate reticulum-Golgi (ERGIC). Lastly, the vesicles that contain the virus particles combine with the plasma membrane to release the virus. ${ }^{(15)}$

\section{The immune response towards}

\section{COVID 19 include:}

A- Antigen presentation in coronavirus infection.

Once the virus reaches the cells, the antigen is introduced to the antigenpresenting cells (APC), which play a basic role in the immunity against the virus. Antigenic peptides are expressed in humans by major histocompatibility complexes (MHC or HLA), and then identified by virus-specific $\mathrm{T}$ lymphocytes (CTLs). Thus, knowledge of the SARS-CoV-2 antigen presentation will increase our knowledge of COVID-19 pathogenesis. SARS-CoV's antigen presentation relies mainly on MHC- I molecules but MHC II also helps in its presentation. The vulnerability to SARS-CoV may be related to several HLA polymorphisms such as HLA-B*4601, HLA-B*0703, HLA-DR B1 * 1202, and, HLA$\mathrm{Cw}^{*} 0801$, whereas, the defense mechanism against has been related to alleles HLA-DR0301, HLA-Cw1502 and, HLA-A*0201 defense mechanism. (16)

The vulnerability to MERS-CoV infection has been contributed to MHC II molecules such as HLA-DRB1*11:01 and HLA-DQB1*02:0. In addition, the gene polymorphisms of $\mathrm{MBL}^{(26)}$ (mannose-binding lectin) which is related to antigen presentation are associated with the possibility of SARS-CoV infection. These findings would give useful ways for COVID-19 diagnosis and prevention. ${ }^{(17)}$

\section{B-Cellular and humoral immunity:}

The immunological pathways initiated against SARS-CoV-2 are mediated by $\mathrm{T}$ cell and $\mathrm{B}$ cell, in addition to direct stimulation of macrophage and stimulated lymphocyte response. Both processes include antigen recognition and presentation. B-cell, T-helper and, cytotoxic $\mathrm{T}$ cells are activated too. Cytotoxic $\mathrm{T}$ cells may kill the virus directly, while helper $\mathrm{T}$ cells present it 
An overview of immunopathogenesis towards COVID-19

Noha Saber Shafik to $\mathrm{B}$ cells for immunoglobulin production and neutralization. (18) SARS-CoV-2 attacks respiratory mucosal epithelial cells and spreads to other cells, infecting white blood cells in the peripheral and immune cells, particularly $\mathrm{T}$ lymphocytes. The cellular response can be identified via multilobular infiltration and lymphopenia observed in SARS-CoV2. Lymphopenia is documented in COVID-19 patients with cellular immune deficiency and suggests an immune response to SARS-CoV-2 mediated by such cells. Damage to lymphocytes by a coronavirus, including $\mathrm{T}$ lymphocytes, leads to lymphopenia, predisposes to secondary bacterial infections, and increases the severity. ${ }^{(19)}$

A decrease in $\mathrm{T}$ helper cells along with regulatory $\mathrm{T}$ cells count was reported in patients with less severe COVID-19 but a pronounced decrease in $\mathrm{T}$ helper cells, regulatory $\mathrm{T}$ cells, and memory $\mathrm{T}$ cells occur in extreme COVID-19. Surprisingly, a higher naïve population of T-helper cells indicated that lymphocyte analysis should be considered for initial COVID-19 patient screening along with the neutrophillymphocyte-ratio (20) An increase in proinflammatory cytokine levels that suggest $\mathrm{T}$ cell-mediated response to SARS-CoV-2, resulting in a cytokine storm that causes hyper-inflammation leading to severe COVID-19 pneumonia. (18) COVID-19 patients showed higher plasma levels of proinflammatory cytokines along with chemokines such as IFN $\gamma$, IL-1, MIP10, and MCP-1, while extreme cases requiring ICU admission had higher TNF- $\alpha$, G-CSF, MCP-1, MIP-10, IL-8, IL-10, and MIP-1A concentrations.
Increased IL-6 expression in serum and IL-2 receptor was documented in severe cases; this may play a crucial role in evaluating COVID-19 severity. ${ }^{(20)}$

In SARS-CoV and MERS-CoV infections, and also in COVID-19, the upregulation of pro-inflammatory cytokines in serum was found associated with significant pulmonary damage and inflammation. Surprisingly, high levels of both Th1 and Th2 cytokines were documented in SARS-CoV-2 infections in comparison to SARS and MERS, where only Th1 cytokines were upregulated. ${ }^{(21)}$

In COVID-19 pathogenesis, the humoral immune response plays a role. When the levels of immunoglobulins in COVID-19 decrease, this may suggest effects on antibody-producing B lymphocyte cells. While SARS-CoV-2 antigens have shown the potential to stimulate the development of antibodies, the impact of general lymphopenia may have caused immunoglobulins to deplete. ${ }^{(19)}$

Antibodies produced against SARSCoV-2, especially anti-spike protein antibodies, may be responsible for immune cell infection. Antibodydependent enhancement (ADE) requiring pre-exposure to particular antigenic coronavirus epitopes may be responsible for the severity of the disease resulting in acute respiratory damage, and other complications such as cellular immunodeficiency, activation of coagulation cascades, myocardial damage, liver, and kidney affection, and superimposed secondary bacterial infection. ${ }^{(2)}$

\section{C) Cytokine storm in COVID- 19:}


SOHAG MEDICAL JOURNAL Vol. 24 No. 2 April 2020
An overview of immunopathogenesis towards COVID-19

Noha Saber Shafik
Acute respiratory distress syndrome is the major cause of death in COVID-19 infected patients. ARDS is a frequently reported event for SARS-CoV-2, SARS-CoV, and MERS-CoV infections. The cytokine storm is one of the key mechanisms for ARDS. In SARS-CoV infection, the unregulated systemic inflammatory reaction results from the release of large quantities of pro-inflammatory cytokines and chemokines by immune-effector cells. In addition, there is high serum levels of IL-6, IFN- $\alpha$, and CCL5, CXCL8, CXCL-10 compared to those with mildmoderate illness. The cytokine storm will initiate a severe attack on the body by the immune system. This will cause ARDS and several organ damages, and eventually death in serious cases of SARS-CoV-2 infection. ${ }^{(23)}$

\section{Mechanism of COVID-19 evasion from the immune system:}

SARS-CoV and MERS-CoV use various methods to suppress immune responses to be still live in host cells. The pattern recognition receptors (PRRs) can identify the pathogenassociated molecular patterns (PAMPs). SARS-CoV and MERS-CoV, however, may stimulate the formation of doublemembrane vesicles that lack PRRs and then replicate in these vesicles, thus preventing their dsRNA's host recognition. IFN-I (IFN- $\alpha$ and IFN- $\beta$ ) has a protective impact on SARS-CoV and MERS-CoV infections but IFN-I pathways are suppressed in the infected mice. ${ }^{(24)}$

MERS-CoV Accessory Protein 4a can block IFN induction by direct combination to double-stranded RNA at the MDA5 activation site. Furthermore, the membrane proteins ORF4a, ORF4b,
ORF5, and MERS-CoV suppress IFN regulatory factor 3 (IRF3) nuclear transport and IFN $\beta$ promoter activation. Coronavirus may also have affected the antigen presentation process. For example, gene expression associated with the antigen presentation is down-regulated after a MERS-CoV infection. Therefore, the destruction of SARS-CoV-2's immune evasion is important in its treatment and specific drug development. ${ }^{(25)}$

\section{Vaccines:}

Effective SARS-CoV-2 vaccines are important to minimize the seriousness of the disease, viral shedding, and transmission, thereby helping to manage outbreaks of coronaviruses. There are several methods for vaccination against SARS-CoV, MERS-CoV tested in primates, including a live attenuated virus, viral vectors, inactivated viruses, subunit vaccines, recombinant DNA, and protein vaccines. ${ }^{(23)}$

These trials are currently in progress but it takes months to years to produce the SARS-CoV-2 vaccines. There could currently be some promising targets for SARS-CoV-2 but further laboratory and clinical data needs to be examined as well. The WHO is cooperating with Chinese scientists to launch more than 80 clinical trials on possible SARSCoV-2 treatment. ${ }^{(23)}$

\section{Conclusion:}

Further studies are needed to understand the immunopathogenesis of SARS-CoV-2. Any immunomodulatory action against $\mathrm{T}$-helper 2 could provide life-saving opportunities for severely ill- patients and reduce the disastrous effects of inflammatory pneumonia. 
SOHAG MEDICAL JOURNAL

Vol. 24 No. 2 April 2020
An overview of immunopathogenesis towards COVID-19

Noha Saber Shafik
One of the therapies is interleukin-10, which inhibits IFN-gamma and IL-12 and TNF-alpha and directs the immune system towards Th2 and away from Th-

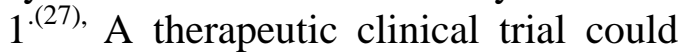
shed light on the impact of such immunomodulatory action on the morbidity and mortality of seriously ill COVID-19 infected patients.

\section{References:}

1-Bassetti M, Vena A, Roberto Giacobbe D. The Novel Chinese Coronavirus (2019$\mathrm{nCoV}$ ) Infections: challenges for fighting the storm. Eur. J. Clin. Invest; 2020; 13209.

2- Zhou P, Yang X, Wang $X$, Hu B, Zhang L, Zhang W, et al. A pneumonia outbreak associated with a new coronavirus of probable bat origin, Nature; 2020; 579 (7798): 270-3.

3-Lee JY, Kim YJ, Chung EH, Kim DW, Jeong I, Kim Y, et al. The clinical and virological features of the first imported case causing MERS-CoV outbreak in South Korea, 2015. BMC. Infect. Dis.; 2017; 17: 498.

4-Perlman $S$ and Netland J. Coronaviruses post-SARS: update on replication and pathogenesis, Nat. Rev. Microbiol. ; 2009; 7: 439-50.

5- Lu R, Zhao X, Li J, Niu P, Yang B, Wu $\mathrm{H}$ et al. Genomic characterization and epidemiology of 2019 novel coronavirus: implications for virus origins and receptor binding. Lancet; 2020; 20: 30251-8.

6- Fehr AR and Perlman S: Coronaviruses: an overview of their replication and pathogenesis. Methods in Molecular Biology.; 2015; 1282: 1-23.

7- Zhu N, Zhang D, Wang W, Li X, Yang B, Song $\mathbf{J}$, et al. A novel coronavirus from patients with pneumonia in China, 2019. New England Journal of Medicine; 2020; 2001017.
8-Wrapp D, Wang N, Corbett KS, Goldsmith JA, Hsieh CL, Abiona Q et al. Cryo-EM structure of the 2019-nCoV spike in the prefusion conformation. Science; 2020; 367 (6483): 1260-3.

9- Hoffmann M, Kleine-Weber H, Kruger N, Muller M, Drosten C, Pohlmann S. The novel coronavirus 2019 (2019-nCoV) uses the SARS-coronavirus receptor ACE2 and the cellular proteases TMPRSS2 for entry into target cells. Bio. Rxiv; 2020; 31: 929042.

10 -Habibzadeh $P$ and Stoneman EK . The Novel Coronavirus: A Bird's Eye View. Int J Occup Environ. Med.; 2020; 11: 65-71.

11- Zhu N, Zhang D, Wang W, Li X, Yang B, Song J, et al. China Novel Coronavirus Investigating and Research Team. A Novel Coronavirus from Patients with Pneumonia in China, 2019. N Engl. J. Med; 2020; 382:727-33.

12- Huang C, Wang Y, Li X, Ren PL, Zhao PJ, Hu Y, et al. Clinical features of patients infected with 2019 novel coronavirus in Wuhan, China, Lancet; 2020; 6736: 30183-5.

13- Millet JK and Whittaker GR. Host cell entry of Middle East respiratory syndrome coronavirus after two-step, furin-mediated activation of the spike protein, Proc. Natl Acad. Sci. U S A; 2014; 111: 15214-19.

14- Kuba K, Imai Y, Ohio-Nakanishi T, Penninger JM. Trilogy of ACE2: a peptidase in the renin-angiotensin system, a SARS receptor, and a partner for amino acid transporters, Pharmacol. Ther.; 2010; 128: 119-28.

15- Wit E, Doremalen N, Falzarano D, Munster VJ. SARS and MERS: recent insights into emerging coronaviruses. Nat. Rev. Microbiol.; 2016; 14: 523-534.

16- Wang S, Chen K, Chen M, Li WY, Chen YJ, Tsao CH et al. Human leukocyte antigen class I Cw 1502 and class II DR 0301 genotypes are associated with 
resistance to severe acute respiratory syndrome (SARS) infection. Viral. Immunol.; 2011; 24: 421-426.

17- Hajeer A, Balkhy H, Johani S, Yousef MZ, Arabi Y, et al. Association of human leukocyte antigen class II alleles with severe Middle East respiratory syndromecoronavirus infection. Ann. Thorac. Med.; 2016; 11: 211-3.

18- Ahmed SF, Qadeer AA, McKay MR. Preliminary Identification of Potential Vaccine Targets for the COVID-19 Coronavirus (SARS-CoV-2) Based on SARS-CoV Immunological Studies. Viruses; 2020; 12: 254.

\section{9-Chen Y, Liu Q, Guo D.Emerging coronaviruses: Genome structure, replication, and pathogenesis. J. Med. Virol.; 2020; 92: 418-23.}

20- Qin C, Zhou L, Hu Z, Zhang S, Yang S, Tao Y, et al. Dysregulation of an immune response in patients with COVID-19 in Wuhan, China Clin Infect. Dis.:2020; 248.

21- Huang C, Wang Y, Li X, Ren L, Zhao J, Hu Y, et al. Clinical features of patients infected with 2019 novel coronavirus in Wuhan, China. Lancet; 2020; 395: 497-506.

22- Tetro JA.Is COVID-19 receiving ADE from other coronaviruses?. Microbes Infect.; 2020; 22: 72-73.
23- Li X, Geng M, Peng Y, Meng L, Lu S. Molecular immune pathogenesis and diagnosis of COVID-19. Journal Pre-proof.; 2020; 20: 30204-5.

24- Channappanavar R, Fehr AR, Zheng J, Wohlford-Lenane C, Abrahante JE, Mack M, et al. IFN-I response timing relative to virus replication determines MERS coronavirus infection outcomes, J. Clin. Invest.; 2019; 130: 3625-39.

25- Menachery V, Schafer A, BurnumJohnson K, Mitchell HD, Eisfeld AJ, Walters KB, et al. MERS-CoV and $\mathrm{H} 5 \mathrm{~N} 1$ influenza virus antagonize antigen presentation by altering the epigenetic landscape, Proc. Natl. Acad. Sci. U. S. A; 2018; 115: 1012-21.

26-Tu X, Chong WP, Zhai Y, Zhang H, Zhang $\mathbf{F}$, Wang $S$, et al. Functional polymorphisms of the CCL2 and MBL genes cumulatively increase susceptibility to severe acute respiratory syndrome coronavirus infection, J. Infect. 71 (2015) 101-109.

27- Abdulamir AS and Hafidh RR. The Possible Immunological Pathways for the Variable Immunopathogenesis of COVID-19 Infections among Healthy Adults, Elderly, and Children. Electronic Journal of General Medicine, 2020, 17(4), em202 